
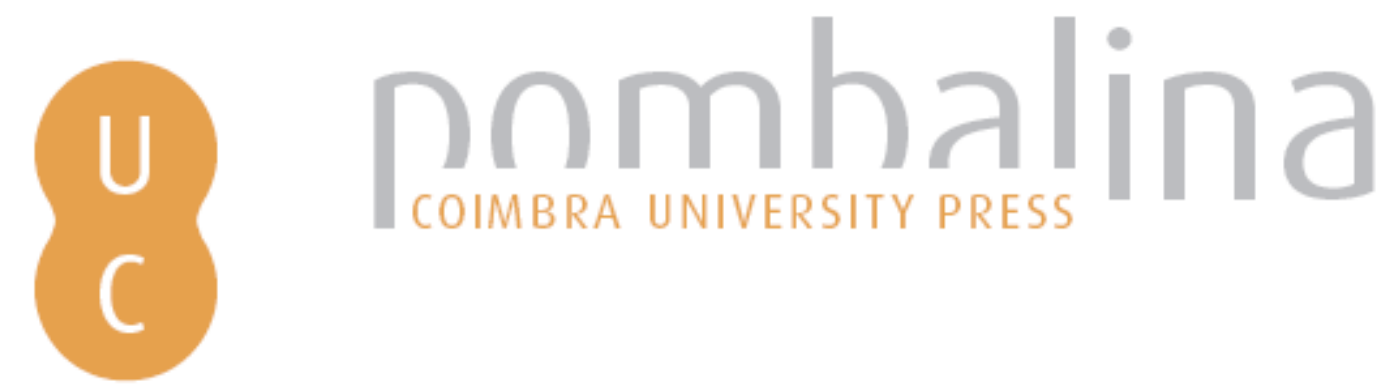

Criterios para el análisis, la valoración y la elaboración de materiales didácticos de
español como lengua extranjera/segunda lengua para niños y jóvenes

Autor(es): Blanco Canales, Ana

Publicado por: Imprensa da Universidade de Coimbra

URL

persistente: URI:http://hdl.handle.net/10316.2/41008

DOI: $\quad$ DOI:http://dx.doi.org/10.14195/978989261231-7_5

Accessed : $\quad$ 26-Apr-2023 16:23:40

A navegação consulta e descarregamento dos títulos inseridos nas Bibliotecas Digitais UC Digitalis, UC Pombalina e UC Impactum, pressupõem a aceitação plena e sem reservas dos Termos e Condições de Uso destas Bibliotecas Digitais, disponíveis em https://digitalis.uc.pt/pt-pt/termos.

Conforme exposto nos referidos Termos e Condições de Uso, o descarregamento de títulos de acesso restrito requer uma licença válida de autorização devendo o utilizador aceder ao(s) documento(s) a partir de um endereço de IP da instituição detentora da supramencionada licença.

Ao utilizador é apenas permitido o descarregamento para uso pessoal, pelo que o emprego do(s) título(s) descarregado(s) para outro fim, designadamente comercial, carece de autorização do respetivo autor ou editor da obra.

Na medida em que todas as obras da UC Digitalis se encontram protegidas pelo Código do Direito de Autor e Direitos Conexos e demais legislação aplicável, toda a cópia, parcial ou total, deste documento, nos casos em que é legalmente admitida, deverá conter ou fazer-se acompanhar por este aviso.

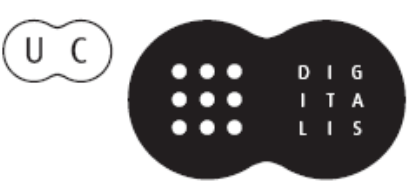




\section{ENSEÑAR ESPAÑOL EN LA ACTUALIDAD \\ CONTRIBUCIONES DIDÁCTICAS}

MARÍA LUISA' AZNAR JUAN ELENA GAMAZO CARRETERO II.

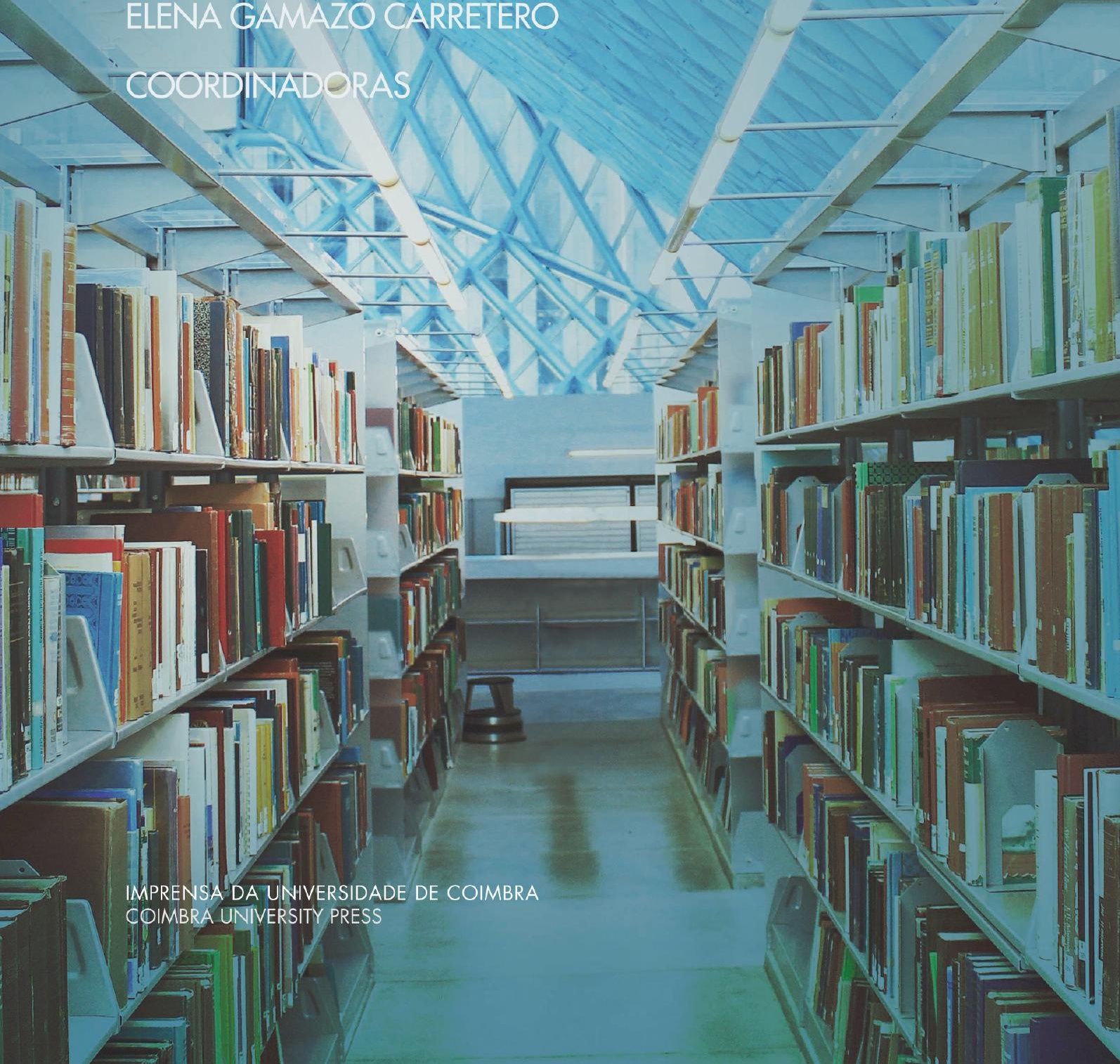




\title{
CRITERIOS PARA ELANÁLISIS, LA VALORACIÓN Y LA ELABORACIÓN DE MATERIALES DIDÁCTICOS DE ESPAÑOL COMO LENGUA EXTRANJERA/SEGUNDA LENGUA PARA NIÑOS Y JÓVENES
}

Ana Blanco Canales Universidad de Alcalá (España)

\begin{abstract}
Spanish's future as a foreign language subject is closely linked to its development in regulated teaching curriculums for elementary and high schools. It is precisely in those two areas where a significant development is expected to happen. Consequently, our aim is twofold: a) to provide authors and editors with reference points for the creation of didactic materials and b) to help teachers, supervisors and educational managers in analysing and assessing already existent materials. For this purpose we have ascertained a number of criteria, based on a critical revision of four key texts in the field of language teaching: Marco Común Europeo de Referência para las lenguas, Orientações curriculares para o ensino médio, National Standards in Foreign Language Education and Plan Curricular from Instituto Cervantes. Thus, using these criteria and the methodological proposals developed by various authors in recent decades as a landmark, we have a created an analytical tool for assessing and creating didactic materials.
\end{abstract}


Keywords: school context, didactic materials, analysis and assessment.

\section{RESUMEN}

El futuro del español como lengua extranjera está estrechamente ligado al desarrollo que adquiera nuestra lengua en los programas reglados de primaria y secundaria, pues son estos los tramos educativos en los que se prevé un aumento más significativo. Con objeto de orientar a autores y editores en la elaboración de material didáctico así como de ayudar a profesores, coordinadores, gestores educativos en el análisis y valoración de los materiales existentes, hemos establecido unos criterios que fundamenten y sirvan de apoyo a las tareas mencionadas. Son el resultado de la revisión crítica de cuatro documentos educativos de enorme relevancia para la enseñanza de lenguas: el Marco Común Europeo de Referencia para las lenguas, las Orientações curriculares para o ensino médio, los National Standards in Foreign Language Education y el Plan Curricular del Instituto Cervantes. A partir de estos criterios así como de propuestas elaboradas por diferentes autores en las últimas décadas, hemos desarrollado una herramienta de análisis encaminada a facilitar tanto la valoración como la elaboración.

Palabras claves: contextos escolares, material didáctico, análisis y valoración.

\section{INTRODUCCIÓN}

Durante la última década, el mercado editorial del español como lengua extranjera (E/LE) ha experimentado un notable desarrollo, 
consecuencia lógica del incremento del número de alumnos que cursan estudios de español. Según los datos proporcionados por el Instituto Cervantes en su Enciclopedia del Español en el Mundo (2006), 14 millones de personas estudian español como lengua extranjera, cantidad que puede aumentar sensiblemente a medio plazo, a medida que la ley del español en Brasil comience a ser efectiva $^{1}$. En este sentido, se calcula que el número de estudiantes en este país pase del millón actual a los 11 millones. De la misma manera, también se prevé un aumento de las cifras en EEUU (en torno al $60 \%^{2}$ ) con lo que se duplicarán los 6 millones con los que cuenta en la actualidad, fenómeno que afectará, sobre todo, a la enseñanza primaria y secundaria, segmentos educativos en los que ya se halla concentrada la mayor parte de la demanda. Las perspectivas en Europa son igualmente halagüeñas, donde, en general, se aprecia un desarrollo expansivo muy fuerte, con un ritmo de crecimiento constante, que afecta, como en los casos anteriores, a las enseñanzas medias.

Cabe imaginar, pues, un futuro próximo en el que la presencia del español en escuelas e institutos de todo el mundo se habrá multiplicado de forma significativa. Será necesario contar con una importante oferta de materiales didácticos entre los que poder elegir aquellos que más se adecuen a la edad y perfil de los estudiantes, sus necesidades formativas, la realidad sociocultural, la situación educativa y las directrices curriculares oficiales. A pesar de que contamos con un volumen creciente de publicaciones para la

\footnotetext{
${ }^{1}$ La Ley 11.161 (Ley sobre la enseñanza del español) fue aprobada por el Parlamento brasileño el 7 de julio de 2005; establece la obligatoriedad de que las escuelas de enseñanzas medias ofrezcan la lengua española como asignatura optativa. Se prevé que habrá un número muy importante de escuelas que incluirá el español en su oferta, pero el futuro es algo incierto dado que todo ello requiere de una importante inversión en recursos (profesorado, material didáctico, etc.) que dificulta la puesta en marcha.

2 Marcos Marín, F. (2006). Español y lengua hispana en los Estados Unidos de América. In Instituto Cervantes. Enciclopedia del Español en el mundo (178-187) Madrid: Círculo de Lectores, IT \& Plaza y Janés.
} 
enseñanza del español, continúan las carencias en lo que respecta al material destinado a niños y jóvenes, precisamente el tramo de edad que más impulso está dando y dará al español.

La proliferación de materiales educativos de E/LE o de español como segunda lengua (E/L2) pero, especialmente, la acuciante necesidad de su elaboración para contextos escolares hace imprescindible el desarrollo de instrumentos de análisis y valoración que:

- faciliten al profesor la selección del más adecuado a su situación educativa, labor que en este caso resulta de más dificultad, habida cuenta de la enorme diversidad con la que nos encontramos y los numerosos factores con que se define cada una de ellas;

- orienten a los autores a la hora de establecer los criterios lingüísticos, didácticos y metodológicos fundamentales para el diseño y desarrollo de materiales escolares;

- permitan a las editoriales detectar vacíos o necesidades y orientar, así, la producción del sector en la dirección más conveniente;

- proporcionen a los investigadores información sobre las formas de implementación y adaptación de las diferentes teorías sobre la lengua y los modelos metodológicos, así como de sus resultados, información que resultará de gran trascendencia para la mejora de los procesos de aprendizaje del español como lengua extranjera.

Por otra parte, no podemos olvidarnos de que el diseño de instrumentos de análisis, selección y elaboración de materiales para la enseñanza de lenguas es uno de los centros de interés de las políticas educativas europeas desde mediados de los años noventa del passado siglo. También lo es el establecimiento de criterios de calidad que sirvan de referencia a los autores para la creación de materiales, 
tarea en la que se ha invertido un gran esfuerzo, como demuestra el número de trabajos disponibles: la guía para autores de materiales que acompaña al documento principal del Marco Común Europeo de Referencia para las lenguas (Hopkins, 2000), las orientaciones de Consejo de Europa para la elaboración de materiales (Fenner \& Newby, 2000 y 2007), el protocolo para la evaluación de materiales y programas elaborado por la asociación AGERCEL (Association de Gestion du Réseau des Centres d'Études des Langues; Agercel, 2000).

\section{Documentos educativos para el establecimiento de los criterios}

La propuesta que presentaremos a lo largo de estas páginas se ha articulado a partir de algunos documentos educativos que se han ido publicando durante la última década con objeto de promover el aprendizaje de lenguas extranjeras y de establecer unas pautas que aseguren la eficacia y calidad del proceso, así como su adecuación a la realidad plurilingüe y multicultural actual. Estos documentos nos han servido para fijar los criterios para el análisis, valoración y elaboración de materiales didácticos que subyacen a nuestra propuesta. El primero de estos documentos ha sido el Marco Común Europeo de Referencia para las lenguas: aprendizaje, enseñanza, evaluación (en adelante, MCER), cuyas directrices -fijadas desde el Consejo Social Europeo- han sido asumidas fehacientemente por las instituciones educativas de los países europeos.

Otro de los documentos que hemos tenido en consideración ha sido el de las Orientações curriculares para o ensino médio. Conbecimentos de lingua estrangeira (espanhol) (2006), texto bastante fiel al MCER en sus planteamientos generales. Nos ha parecido pertinente su uso, dada la repercusión que se prevé que tenga la ley del español en Brasil. En el documento se advierte sobre 
la necesidad de contar con análisis rigurosos del material disponible, para lo que se necesitan, previamente, propuestas amplias que recojan las múltiples dimensiones a las que atender en el proceso de enseñanza-aprendizaje.

Hoje, no Brasil, encontrasse grande oferta de livros didáticos [...]. Contudo, uma análise desses materiais pautada em critérios claramente definidos e objetivos torna-se imprescindível, como aponta Melone (2000: 234), para que eles, de fato, atendam às necessidades específicas de cada situação de ensino e, nesse caso, aos objetivos educacionais do ensino médio. (p. 154)

Nos hemos valido también del documento norteamericano National Standards in Foreign Language Education Project (1996), dada su importancia e influencia para la enseñanza de lenguas extranjeras en EEUU. Las diferentes instituciones educativas federales han asumido las recomendaciones vertidas en él, de manera que, en la actualidad, tanto los currículos educativos como la labor docente se guían por los cinco macroobjetivos marcados. No se trata de una guía curricular en la que se especifican objetivos, contenidos, metodología, etc., sino de una propuesta de líneas de actuación que afecta a profesores, gestores educativos, familias, editores, autores y empresarios.

The standards are not a curriculum guide. While they suggest the types of curricular experiences needed to enable students to achieve the standards, and support the ideal of extended sequences of study that begin in the elementary grades and continue through high school and beyond, they do not describe specific course content, nor recommended sequence of study. They must be used in conjunction with state and local standards and curriculum frameworks to determine the best approaches and 
reasonable expectations for the students in individual districts and schools. (Executive Sumary of Standards for foreign language learning: Preparing for the 21 st century, 3)

Por último, la propuesta responde, lógicamente, a las indicaciones del Instituto Cervantes recogidas en su Plan Curricular, pues es el documento de referencia más importante con el que contamos para la enseñanza del español. Los Niveles de referencia para el español - una parte del renovado Plan Curricular - (como no caso anterior) recogen las aportaciones fundamentales de la investigación en lingüística aplicada a la enseñanza de lenguas de los últimos años; asimismo, se han desarrollado de acuerdo a los planteamientos del Departamento de Política Lingüística del Consejo de Europa.

\section{Criterios del MCER}

La herramienta de análisis que hemos diseñado se ha perfilado sobre la base de las reflexiones del MCER relativas a los diferentes componentes y dimensiones que intervienen en todo proceso de enseñanza/aprendizaje de una lengua extranjera, a su concepción sobre lo que supone el uso de una lengua (desde un punto de vista cognitivo, comunicativo y sociocultural) y a las competencias que debe desarrollar el alumno. Asimismo, hemos tratado de que la propuesta dé respuesta a tres aspectos concretos: a) presencia, tratamiento y función de las tareas, b) carácter dinámico y reflexivo del aprendizaje y c) desarrollo de estrategias de aprendizaje y fomento de la autonomía.

Como es bien conocido, el MCER entiende que la enseñanza de lenguas debe estar orientada a la acción, pues considera que cualquier alumno es un usuario de la lengua que está aprendiendo, y como usuario, es un agente social, es decir, un miembro de una sociedad dentro de la cual se relaciona y en la que interactúa verbalmente con 
el objeto de realizar tareas. En este sentido, todo acto de aprendizaje o de enseñanza de idiomas estaría relacionado con cada una de las dimensiones que entran en juego en la comunicación: las estrategias, las tareas, los textos, las competencias generales, la competencia comunicativa, las actividades de lengua, los procesos, los contextos y los ámbitos. Nos parece, por tanto, conveniente analizar de qué manera se contemplan y reflejan todas ellas en los materiales didácticos.

Ya que la tarea es el principio que articula el proceso de enseñanza, resulta necesario aclarar cuáles son las bases sobre las que se apoya el concepto, si queremos ver su tratamiento en los materiales didácticos. Por una parte, hay que destacar que toda tarea supone la puesta en práctica de unas competencias dadas a fin de realizar un conjunto de acciones en un ámbito determinado, con una finalidad concreta y con un resultado. Por otro lado, la tarea pretende implicar al alumno en una comunicación real, tiene un sentido - habla, lee, escribe, escucha para algo -, es factible y tiene un resultado identificable. Asimismo, proporciona todos los elementos (lingüísticos, contextuales, culturales...) y las razones para llevar a cabo un proceso de comunicación, lo que la convierte en marco idóneo para un aprendizaje significativo, que favorezca la construcción de conocimiento y que incluya las experiencias personales de los alumnos como elementos fundamentales del proceso. En el análisis de materiales que llevemos a cabo habrá de comprobarse si se cumplen estas condiciones, y, en caso contrario, cuál es la concepción y funcionalidad de la tarea.

El MCER defiende un aprendizaje dinámico y participativo, que dé la oportunidad a los alumnos de descubrir de forma autónoma los contenidos; que haga uso de técnicas inductivas y deductivas en lugar de las meramente memorísticas; que desarrolle actividades creativas, dependientes de actos comunicativos. Todo ello está estrechamente relacionado con otra de las ideas básicas del MCER: la de favorecer el desarrollo de estrategias de aprendizaje y, por ello, 
promover la autonomía como aprendices. Para que esto sea posible, se deben crear contextos adecuados que les den oportunidad para reflexionar sobre la lengua y sobre el propio proceso de aprendizaje, así como de autoevaluar los logros que van siendo alcanzados, identificar las debilidades y fortalezas y planificar la mejora. En el contexto educativo en el que nos hallamos - enseñanza reglada - este planteamiento adquiere una relevancia enorme, como se puede apreciar por los currículos oficiales, por lo que hemos considerado que tales criterios han de incluirse en el análisis.

\section{Criterios de las Orientações Curriculares Para O Ensino Médio}

De todas las cuestiones que se abordan en las Orientações a propósito de la enseñanza de lenguas y, concretamente, del español, nos ha parecido de especial relevancia para nuestros fines tres de ellas: las lenguas extranjeras en contextos escolares reglados, el papel de la lengua materna y la diversidad lingüística y cultural del español. Hemos tratado de que en nuestra herramienta de análisis haya lugar para valorar cómo se resuelven o tratan todas ellas y, así, atender algunas de las máximas preocupaciones de los responsables educativos brasileños.

Con respecto a la primera cuestión, no cabe duda de que el contexto en el que tiene lugar el proceso de aprendizaje de la lengua determina el papel que va a desempeñar su enseñanza, y así aparece señalado desde el principio en las Orientações (2006). En este sentido, se insiste en que hay diferencias muy importantes entre el ámbito de la educación regular y el de la enseñanza libre, pues en la primera, la enseñanza de lenguas extranjeras no puede ser entendida como un fin en sí misma, sino que debe integrarse con otras disciplinas curriculares y buscar convergencias e 
interdependencias, pues forma parte de un todo. Por esta razón, parece primordial trabajar las lenguas extranjeras no solo como formas de comunicación, sino también como constructos portadores de significados, conocimientos y valores, de manera que se cumpla, así, con los cuatro objetivos marcados por la UNESCO como ejes estructurales de la educación: aprender a conocer, aprender a hacer, aprender a vivir y aprender a ser (p. 131).

Por lo que respecta a la cuestión del papel de la lengua materna - el portugués - en la enseñanza y aprendizaje del español, no es de extrañar el interés que genera, dada la similitud entre ambas, similitud que facilita el primer acercamiento pero que dificulta el avance y la superación de errores. Se defiende en todo momento la adopción de una perspectiva contrastiva que conduzca a una reflexión interlingüística y, a partir de ahí, a un mejor conocimiento del funcionamiento de las dos lenguas. Esta reflexión y comparación debe constituir una práctica habitual y seria, y no debe limitarse a aspectos léxicos (los menos relevantes), sino que ha de atender a otras de tipo fonético, morfosintáctico, pragmático e, incluso, discursivo.

Por último, la heterogeneidad del español y su cultura es también objeto de preocupación, puesto que se considera inaceptable adoptar planteamientos centralistas y reduccionistas. Así, la conocida pregunta “¿qué español enseñar?” debe sustituirse por otra: “cómo enseñar una lengua tan plural y heterogénea, sin sacrificar sus diferencias ni reducirlas a meras anécdotas?". En este sentido, se insiste mucho en que los materiales didácticos recojan las variedades del español en sus contextos sociales y culturales y trasmitan realidades complejas, en lugar de limitarse, como viene siendo habitual, a simples listas de curiosidades.

Continuar considerando a las variedades lingüísticas y culturales latinoamericanas del español como conjuntos estables de creencias, valores y comportamientos, que pueden agregarse como atractivos 
complementos del material didáctico lleva a un divorcio de la lengua con su contexto cultural y social. (Buge, 2000 apud Orientações, 2006, p. 137).

\section{Criterios del National Standards In Foreing Education Project}

El documento National Standards surgió bajo la iniciativa del gobierno federal de los EEUU ante la preocupación por el escaso interés que la sociedad mostraba por el aprendizaje de lenguas extranjeras. De manera similar al MCER, pretende ser un marco general para el desarrollo de currículos y programas de enseñanza. Aunque las pautas no son propiamente directrices, sino metas que se han de lograr, tienen también la intención de orientar al profesor en la propia actividad docente en las aulas.

En el documento se especifican los conocimientos y habilidades que los alumnos deben haber adquirido al final de la enseñanza secundaria. Todos ellos se hayan estructurados en torno a cinco macroobjetivos o metas, cada una de las cuales se divide, a su vez, en dos o tres pautas o conjuntos de tareas que han de saber realizar. Las metas son:

- Culturas: adquirir conocimientos y una mayor comprensión de otras culturas.

- Conexiones: hacer conexiones con otras asignaturas.

- Comparaciones: desarrollar conocimientos sobre el carácter de las lenguas y las culturas.

- Comunidades: relacionarse con comunidades multilingües dentro y fuera del país.

Ofrece, además, una serie de reflexiones sobre siete elementos curriculares: la lengua como un sistema pluridimensional, las estrategias 
de comunicación, los contenidos culturales, las estrategias de aprendizaje, los contenidos de otras asignaturas, el análisis contrastivo y las habilidades de pensamiento y, por último, el uso de recursos tecnológicos.

Es fácil apreciar las notables semejanzas con el MCER en cuanto a los principios subyacentes. Sólo hay dos aspectos que no aparecen explícitamente en el MCER y sí en el National Standards, hecho que se explica por los diferentes ámbitos de actuación: el MCER es de carácter generalista, mientras que el National Standards se circunscribe a enseñanzas primarias y secundarias regladas. El primero de estos aspectos diferenciadores tiene que ver con la necesidad de establecer conexiones con otros ámbitos del currículo escolar. Se pretende que el alumno pueda reforzar y ampliar conocimientos propios de otras asignaturas a través del idioma extranjero, así como que pueda adquirir información e identificar puntos de vista que solo se pueden captar a través de una lengua y su(s) cultura(s) ${ }^{3}$.

Este planteamiento tiene que ver con lo que se ha llamado aprendizaje por contenidos y currículos integrados, que goza en la actualidad de la confianza y el interés de muchos expertos en metodología y educación. Según Spanos (1989, p. 228), cuando hablamos de integración de lenguaje y contenidos nos estamos refiriendo a tres situaciones: introducir los contenidos de las materias curriculares en la clase de lengua; hacer las clases del resto de las asignaturas más sensibles a los aspectos lingüísticos; o hacer ambas cosas simultáneamente. En este sentido, el enfoque basado en contenidos representa una propuesta que integra la asignatura de lengua (materna o extranjera) con el resto de las áreas curriculares dentro

${ }^{3}$ En las Orientações curriculares del ministerio brasileño también se reflexiona sobre la conveniencia de fomentar un conocimiento integral, en el que las distintas áreas del currículo se hallen interrelacionadas. 
de una perspectiva constructivista y globalizadora del aprendizaje (Trujillo, 2005).

Dado el interés y desarrollo actual de estas teorías ${ }^{4}$, nos ha parecido muy conveniente incluir en nuestra propuesta un apartado en el que identificar si se trabajan o no contenidos de otras áreas del currículo, y en caso de que así sea, qué áreas y qué temas. Se analiza también la función de esos contenidos en el desarrollo de las unidades didácticas, la sistematicidad de la integración, la finalidad, etc.

El segundo aspecto diferenciador entre los dos documentos educativos es de carácter instrumental y se refiere a la necesaria presencia de recursos tecnológicos en las aulas de lenguas extranjeras, dado el enorme potencial que ofrecen de conocimiento de otras realidades y como fuente para obtener material. Actualmente, está generalizado el uso de herramientas multimedia y tecnológicas en las aulas de primaria y secundaria; sin embargo, el material didáctico solo recientemente empieza a incluir como complemento y apoyo cederrones con actividades interactivas, espacios web de aprendizaje desarrollados por las editoriales como apoyo en Internet al libro, enlaces web, etc. La propuesta también intenta comprobar en qué medida el material didáctico ofrece al alumno la posibilidad de aprender con recursos modernos, sin duda, más variados, de mejor calidad técnica y más motivadores.

\footnotetext{
${ }^{4}$ Véase Arnau, J. (2001). La enseñanza de la lengua extranjera a través de contenidos: principios e implicaciones prácticas. Actas del Congreso Internacional. Oviedo: Anaya - MEC; Madrid, D. \& E. García Sánchez, E. (2001). Content-based Second Language Teaching. In E. García Sánchez (Ed.), Present and Future Trends in TEFL (101-134). Almería: Secretariado de Publicaciones de la Universidad de Almería; Met, M. (1994). Teaching content through a second language. In F. Genesee (Ed.), Educating Second Language Children: The whole child, the whole curriculum, the whole community (159-182). New York: Cambridge University Press; Trujillo Sáez, F. (2003). Elements for a redefinition of TEFL in Spanish Secondary Education. In G. Luque Agulló, A. Bueno González \& G. Tejada Molina (Eds.), Las lenguas en un mundo global (101-111). Jaén: Universidad de Jaén.
} 


\section{Criterios del Plan Curricular del Instituto Cervantes}

La propuesta que presentamos en estas páginas está igualmente ligada a las directrices marcadas en el Plan Curricular del Instituto Cervantes (concretamente, en los Niveles de referencia para el español). De él hemos tomado, sobre todo, las categorías para la organización de los objetivos, el tratamiento de los aspectos culturales, socioculturales e interculturales y la dimensión del aprendizaje de la lengua.

Para el establecimiento de los objetivos generales, el Plan Curricular parte de considerar al alumno desde tres dimensiones o perspectivas complementarias, y así, habla del alumno como agente social, como hablante intercultural y como aprendiente autónomo. Cualquier alumno de una lengua extranjera es, como ya vimos más arriba, usuario de esa lengua y como tal, miembro de una sociedad en la que realiza actividades lingüísticas y no lingüísticas. Debe ser capaz de interactuar con los otros miembros y satisfacer sus necesidades y objetivos a través de la comunicación. Por otra parte, como hablante intercultural, ha de reconocer los aspectos relevantes de la nueva cultura y "desarrollar la sensibilidad necesaria para establecer puentes entre la cultura de origen y la cultura nueva». (Plan Curricular, V. I., p. 33). Para ello, el alumno debe disponer de una serie de conocimientos sobre referentes culturales y normas y convenciones sociales, pero sobre todo, ha de mantener una actitud abierta y receptiva que le permita valorar la diversidad cultural como fuente de enriquecimiento personal. Por último, el alumno ha de ser capaz de tomar conciencia de su aprendizaje, tanto en cuestiones afectivas como cognitivas y responsabilizarse de su propio proceso, siendo cada vez más autónomo y desarrollando estrategias propias que le permitan aprender en cualquier situación y a lo largo de toda su vida.

Desde nuestro punto de vista, uno de los mayores logros de los Niveles de referencia es la inclusión del componente de aprendizaje con su correspondiente inventario de procedimientos. De acuerdo una 
vez más con el MCER, el Instituto Cervantes se muestra claramente partidario de los nuevos enfoques psicopedagógicos que consideran que el desarrollo de estrategias de aprendizaje por parte del alumno es una garantía de éxito y de autonomía. Se parte de la idea de que en todo proceso de aprendizaje se activa una serie de estrategias que hacen posible la adquisición de nuevos conocimientos, su almacenaje, su uso y actualización, su interrelación con otros saberes, etc. Si, desde la reflexión guiada y sistemática, el alumno toma conciencia de esas estrategias podrá orientar su aprendizaje en la dirección de sus necesidades, así como mejorarlo y rentabilizarlo. Se trata de aprender a aprender, lo que implica la capacidad de reflexionar en la forma en que se aprende y actuar en consecuencia, autorregulando el propio proceso de aprendizaje mediante el uso de estrategias flexibles y apropiadas que se transfieren y adaptan a nuevas situaciones.

\section{Propuesta de análisis de materiales didácticos en E/LE}

En las últimas cuatro décadas se han ido elaborando distintas propuestas de análisis de materiales, cada una con unos objetivos y fines determinados. La primera en el caso del español fue la de Ezquerra (1974), propuesta que desarrolló con el objeto de poder cumplir con el encargo de la Asociación Europea de Profesores de Español, y que consistía en recoger información sobre los manuales que se estaban utilizando en Europa para la enseñanza de nuestra lengua, más concretamente, sobre los planteamientos didácticos y metodológicos en los que se apoyaban esos manuales. Ezquerra propone una herramienta de valoración crítica encaminada a satisfacer dos objetivos: por un lado, evidenciar de manera clara los contenidos que se estudian en cada libro, y por otra, comprobar si los planteamientos metodológicos defendidos por los autores coinciden con los que realmente plasman, esto es, valorar la coherencia. Su 
propuesta es una guía general de análisis, pensada sobre todo para manuales de corte estructural, que eran los que se estaban utilizando en aquellos años. Esta guía se articula en tres bloques. El primero de ellos se centra en la descripción de los elementos y estructura del manual; el segundo atiende a los principios metodológicos y el último revela los contenidos léxicos y gramaticales al tiempo que valora algunas cuestiones de didáctica.

La segunda propuesta significativa de análisis de materiales la encontramos en EEUU, más de una década después. Se trata del trabajo de Arizpe \& Aguirre (1987), orientado al estudio de referencias culturales y, más concretamente, al tratamiento de elementos cubanos, puertorriqueños y mejicanos. Analizan cuatro aspectos en manuales de E/LE de primer curso de universidad: incorrecciones, estereotipos, simplificaciones excesivas y omisiones.

En 1990 aparece el trabajo de Ramírez \& Hall, quienes desarrollan muy ampliamente toda la cuestión de los contenidos socioculturales, la contextualización de la lengua y la integración de elementos culturales en la enseñanza de la lengua en libros de texto de E/LE utilizados en EEUU. Analizan el contenido cultural desde una triple perspectiva: sociocultural (representación de temas, países y grupos hispanos), sociolingüística (temas de comunicación, situaciones en las que se produce la comunicación y contenidos lingüísticos en relación a las funciones comunicativas) y curricular (componentes de las lecciones).

También de 1990 es la propuesta de Salaberri Ramiro, aunque esta con fines algo diferentes a los de los trabajos anteriores, pues pretende ser una guía que ayude al profesor en la tarea de seleccionar los manuales más adecuados a sus alumnos. La propuesta se plantea a modo de cuestionario organizado en diez apartados: necesidades de los alumnos, objetivos, syllabus, metodología, contenido lingüístico, gradación del lenguaje, repaso, fases dentro de una unidad y destrezas, material de apoyo y otras cuestiones. 
Fernández López (1994) elabora la primera propuesta global para el análisis de materiales didácticos en España. La segunda versión de esta propuesta, de 2004, ofrece algunas mejoras al incorporar dimensiones y aspectos no contemplados en la anterior. Se trata de algo más que un instrumento de análisis, pues su diseño deja ver una profunda reflexión sobre la tarea de la enseñanza de una lengua extranjera. Elabora dos fichas distintas con objeto de poder analizar tanto manuales como materiales. Ambas constan de cuatro grandes apartados: descripción externa, descripción interna, análisis y observaciones. En la descripción externa, tanto para manuales como para materiales, se recoge información bibliográfica y datos sobre el tipo de soporte. En cuanto a la descripción interna, se realiza básicamente a partir de la información que los propios autores y editores proporcionan en los prólogos o presentaciones. En el caso de los manuales se atiende a cuestiones como los objetivos, la metodología, la organización en niveles, el destinatario, la programación que ofrece y la organización de cada lección. En los materiales, se recogen datos sobre objetivos, destrezas, nivel, destinatario, organización del material y organización de cada unidad. El tercer apartado de las fichas va encaminado a analizar de manera exhaustiva y crítica la obra. Es, sin duda, la parte más compleja e interesante. Para el análisis de manuales, la autora propone atender a seis aspectos: el papel de la L1, la presentación de la L2, los contenidos comunicativos, los contenidos lingüísticos, los contenidos culturales, materiales de evaluación. En el caso de los materiales, el análisis se lleva a cabo sobre los contenidos. Fernández López proporciona en las fichas temas concretos sobre los que habrá que investigar en cada caso: contextualización de la lengua, orientación deductiva/inductiva; lengua escrita/lengua oral, presentación, programación, ejercitación y progresión de los contenidos, etc. Gracias al estudio de todos estos aspectos, podremos conocer los fundamentos metodológicos y didácticos subyacentes. 
Otro paso muy importante en el diseño de herramientas de análisis de materiales se da con el trabajo de Areizaga Orube (1997), ya que incorpora como objeto de interés los factores contextuales. La autora está convencida de que en los manuales no solo se proyectan principios sobre la concepción de la lengua y su aprendizaje, sino también los factores contextuales en los que se elaboran y utilizan, por lo que propone un esquema de estudio con dos grandes parámetros: factores contextuales (características de los alumnos y de los profesores, marco de instrucción, etc.) y características metodológicas en relación con los factores contextuales (papel de la primera lengua), orientación contrastiva, orientación del modelo dialectal de lengua y del componente cultural, etc.).

La propuesta más reciente y sofisticada para el análisis y evaluación de materiales didácticos es la de Ezeiza Ramos (2008). Lleva a cabo una profunda revisión de los criterios y procedimientos que se han seguido en los trabajos sobre análisis y evaluación de materiales didácticos, desde la década de 1970 hasta la actualidad. El objetivo de esta investigación es elaborar un modelo general de síntesis para el estudio de materiales, lo que le ha permitido, a su vez, desarrollar una herramienta informática con la que obtener información diversa sobre la configuración metodológica desde criterios lingüísticos, psicolingüísticos y de organización.

Lo que tienen en común la mayoría de estas propuestas es que están pensadas para análisis de materiales destinados a adultos, lo que dificulta o imposibilita su utilización para el caso de estudiantes infantiles o juveniles. La situación de los materiales para su utilización en colegios e institutos resulta mucho más compleja, porque cada etapa, cada nivel educativo, cada edad e, incluso, cada comunidad sociocultural requiere de un libro de texto distinto, que, además, debe ajustarse a unos planteamientos curriculares determinados contemplados en las leyes educativas de cada país. La especificidad de este tipo de situaciones educativas hace aconsejable desarrollar 
instrumentos de análisis propios que permitan describir y valorar otros aspectos relevantes y significativos para estos contextos.

\section{Nuestra propuesta}

Hemos tratado de que nuestra propuesta sea, en sí misma, portadora de los criterios presentados en estas páginas, esto es, que al tiempo que sirve como instrumento-guía, informe con transparencia de los fundamentos metodológicos y didácticos actuales sobre la enseñanza de lenguas extranjeras. La hemos estructurado en ocho bloques más un apartado final a modo de cierre y conclusiones.

Con objeto de simplificar al máximo el análisis del material didáctico por parte de los posibles usuarios, hemos dividido cada apartado en tantos subapartados o aspectos como parece necesario tener en consideración. Se trata de un análisis muy dirigido, en el que se proporciona una serie concreta de puntos sobre los que trabajar, lo que permite homogeneizar los resultados.

El primero de los apartados, el de los datos identificativos, busca simplemente recoger información bibliográfica. Además de las referencias habituales (autor, título, editorial, año, etc.) hemos incluido la de "Otras obras del autor/es", pues, en ocasiones, puede anticiparnos y aclararnos muchos aspectos claves de la obra (planteamientos metodológicos, perspectiva sobre el aprendizaje/ enseñanza de lenguas, etc.).

Nos encontramos a continuación con el apartado dedicado a los aspectos formales del manual: elementos que componen la obra y sus características, edición y estructura y organización del manual. En los dos primeros casos - elementos y edición - (como en el caso anterior), nos parece de especial interés la valoración de la funcionalidad, pues ello nos informará sobre la coherencia del manual y su aspecto global e integrador. Si las ilustraciones o fotografías, por ejemplo, no cumplen 
una función didáctica (introducir, contextualizar, presentar, soportar contenidos) serán un mero adorno y un motivo de distracción que fragmentará el conjunto. Resulta igualmente muy importante analizar la adecuación del material o de los elementos al público al que va destinado, a su edad, origen, nivel de lengua, etc. Mucha atención deberemos también poner a la hora de valorar la imagen que se trasmite (moderna, anticuada, tolerante, democrática, igualitaria, inclusiva, multicultural...) y la diversidad o no sociocultural (¿se representan los diferentes grupos sociales, étnicos, culturales que conforman la comunidad?).

Por lo que respecta a la estructura, además de conocer qué material se proporciona junto con las unidades de trabajo (programación, apéndices, glosarios, etc.) es conveniente conocer qué tipo de estructura presentan esas unidades, pues esto está estrechamente relacionado con la concepción de la lengua y del aprendizaje que subyace. Así, una unidad organizada en secuencias de aprendizaje (presentación, reflexión, práctica dirigida, práctica semidirigida, actividad de comunicación libre) refleja una visión más comunicativa e integradora de la lengua que otra dividida por niveles internos (vocabulario, gramática, fonética). Pero al mismo tiempo, la primera resultará, desde el punto de vista del aprendizaje, más compleja de seguir para el alumno, con la consiguiente incertidumbre que ello suele ocasionar. Lo deseable sería que el analista no se limitara a un simple registro de datos, a una descripción objetiva, sino que proporcionara una valoración crítica justificada.

También en este bloque se estudia la forma de secuenciación de cada apartado concreto, pues es el rasgo que más denota la existencia de un plan claro y consciente de aprendizaje. Se trata de ver la relación que existe entre las distintas actividades, la naturalidad o no con que se pasa de una a otra, su pertinencia dentro de ese bloque, etc.

El tercer apartado de nuestra propuesta se focaliza en la situación educativa con objeto de registrar una serie de datos fundamentales como son la edad de los destinatarios; el nivel de lengua, según las 
referencias del MCER; la duración, es decir, la cantidad de tiempo que se requiere para su completa realización y finalmente, el contexto y la situación educativa. Se trata de señalar si es un manual destinado a enseñanza reglada o no reglada, y en caso de enseñanza reglada, el nivel educativo para el que se ha elaborado (infantil, primaria, secundaria, bachillerato). El hecho de destinarse a programas reglados determinará muchas de las características que debe tener, desde la duración (un año académico) hasta principios básicos curriculares propios de la etapa educativa. De la misma manera, será conveniente especificar el tipo de programa, esto es, si es asignatura obligatoria u optativa, si se halla en una situación de enseñanza bilingüe, si es lengua extranjera o segunda lengua...

De la descripción de los objetivos generales se ocupa el cuarto apartado. Tal y como señalamos más arriba, hemos tomado de los Niveles de referencia para el español del Instituto Cervantes la categorización y la subdivisión interna, con objeto de orientar y facilitar al analista la tarea de identificar los objetivos, y a los autores, la de fijar las metas que deben perseguir. A las tres dimensiones del alumno que se describen en este documento, hemos añadido una cuarta: el alumno como estudiante de un programa de enseñanza reglada, pues en este caso, los currículos oficiales presentan una serie de objetivos transversales, formulados a modo de competencias básicas, que deben estar presentes en todas las asignaturas, incluida la de lengua extranjera. Para la propuesta de análisis, hemos seleccionado aquellas que se prestan más a ser trabajadas en una asignatura de lengua extranjera y que no están contempladas explícitamente en esta asignatura.

Nuestra ficha continúa con el análisis de los principios metodológicos que rigen la obra. Proponemos una serie de dimensiones sobre el aprendizaje con objeto de que se compruebe si están presentes y en qué medida. En su mayor parte, proceden de los documentos educativos y curriculares que hemos manejado y vienen a ser una síntesis de las investigaciones actuales sobre metodología de 
la enseñanza de lenguas extranjeras. Algunas de estas dimensiones están relacionadas con la naturaleza del aprendizaje; otras tratan de valorar la orientación de la enseñanza; también se atiende a la manera de presentar y trabajar los contenidos (contextualizados, integrados); o se centran sobre la concepción de la lengua y el tipo de actividades para su práctica. El apartado termina con un espacio para las observaciones, pues entendemos que, en este caso, es muy difícil marcar simplemente una casilla, y con seguridad, será necesario aclarar el tratamiento que recibe cada uno de los puntos analizados.

En el bloque sexto proponemos una reflexión sobre las formas en que habrá de trabajar el alumno, y está dividido en dos partes. La primera de ellas está dedicada a recoger la tipología de actividades presente en el manual. Sería conveniente, además, registrar en qué lugar de la secuencia, con qué nivel de sistematicidad y valorar el grado de eficacia. No es esta una tarea en absoluto fácil, pues, en muchas ocasiones, las actividades cumplen varias funciones (y se encuadrarían, por tanto, en varias casillas), y en otras, bajo la apariencia de una actividad de comunicación no hay más que un ejercicio estructural. La segunda parte - mucho más fácil - da cuenta de las dinámicas de trabajo: individual, en parejas, en grupos... Además de un mero registro de las formas de realizar las tareas y sus frecuencias, resultaría interesante valorar la conveniencia, la viabilidad y la pertinencia.

A continuación, pasamos a estudiar los componentes del manual, concretamente los enunciados, los textos (orales y escritos) y las fichas. Para ello, hemos creado seis subapartados. El primero corresponde a las instrucciones de trabajo, esto es, a los enunciados. Se trata de indicar sus características más visibles: lengua en que aparecen (L1 o L2), claridad y precisión, longitud y dificultad para su comprensión, correspondencia con el nivel de lengua del material, desempeño de otras funciones al margen de la instrucción (enlazar, cohesionar), etc. Los cuatro siguientes subapartados se refieren a los textos, y en ellos 
se analizan tanto los tipos que podemos encontrar, como la funcionalidad. Esta última cuestión no siempre resulta fácil de describir, pues, como en el caso de la tipología de actividades, en ocasiones no se aprecia con claridad la misión que cumple el texto (tanto porque puede cumplir varias, como porque no se aprecia ninguna de las contempladas). Finalmente, el subapartado sexto se ocupa de las fichas. Se trata de analizar el tipo de información que recogen, la frecuencia con que aparecen y la ubicación (si disponen o no de un lugar propio), la función que cumplen (presentar contenidos, sistematizar, aunar toda la información, cerrar la unidad didáctica...) y el formato (especialmente, en los casos en los que existen distintos tipos de fichas en función de la información que presentan).

El último de los bloques es el de la especificación de los contenidos del material. Es el apartado que más trabajo requiere pero no el más complejo, pues se trata solo de identificar y registrar todos los contenidos que se van tratando a lo largo del material. Puede ser de gran ayuda el cuadro de programación que normalmente acompaña a los libros didácticos. No obstante, siempre es conveniente comprobar que, efectivamente, se trabajan, así como si existen otros que no aparecen en el cuadro (es difícil recoger en una o dos hojas todos los contenidos). Hemos organizado este gran apartado en seis partes. La primera se centra en lo que hemos denominado "contenidos de comunicación”, pues presta atención a los aspectos nociofuncionales de la programación y a los contextos y las acciones concretas de comunicación que se trabajan en las diferentes unidades didácticas. Por ello, este apartado se subdivide, a su vez, en tres. En el primero, se pide anotar los ámbitos nocionales de carácter específico que se hallan presentes, y que son los que permiten al autor seleccionar, secuenciar y graduar los contenidos, así como integrarlos y conferirles unidad y coherencia. El Plan Curricular del IC establece un total de 20 posibles ámbitos: dimensión física del individuo; dimensión perceptiva y anímica; identidad personal; relaciones personales, 
alimentación, educación, trabajo, ocio, información y medios de comunicación; vivienda; servicios; compras, tiendas y establecimientos; salud e higiene; viajes, alojamiento y transporte; economía e industria; ciencia y tecnología; gobierno, política y sociedad; actividades artísticas; religión y filosofía y geografía y naturaleza. Podemos partir de este repertorio e ir comprobando cuáles se han incorporado y qué otros no. En el segundo subapartado, hay que enumerar las situaciones de comunicación e interacción que se dan dentro de los ámbitos señalados en el punto anterior. Así, por ejemplo, en el ámbito "salud e higiene", podríamos encontrar: diálogos en el médico, explicación sobre hábitos de vida saludables, encuesta sobre higiene bucal... El tercero viene a ser una especificación del segundo, pues consiste en anotar las funciones lingüísticas que se trabajan - y que lógicamente están relacionadas con las situaciones de comunicación y con el ámbito nocional -. Continúa el bloque ocho con el registro de los contenidos lingüísticos y el de los contenidos de otras áreas curriculares, en caso de que los haya (matemáticas, literatura, sociales, física, química...). El apartado siguiente se refiere a los referentes culturales, y que, tal y como propone el Instituto Cervantes, hemos agrupado en tres, según estén relacionados con conocimientos generales de países hispanoamericanos, con hechos y personajes de relieve pasados y presentes o con manifestaciones culturales. A continuación - quinta parte - nos centramos en los saberes y comportamientos socioculturales, que hacen referencia «al conocimiento, basado en la experiencia, sobre el modo de vida, los aspectos cotidianos, la identidad colectiva, la organización social, las relaciones personales, etc.» (Niveles..., p. 399). Se trata de un apartado muy amplio y diverso en el que caben todos aquellos aspectos que tengan que ver con las costumbres y formas de vida propias de un país, como pueden ser los horarios, días festivos, la estructura familiar, convenciones sociales para diferentes situaciones, etc. Por último, proponemos recoger los contenidos para el desarrollo en el 
alumno de un aprendizaje estratégico que aparezcan y se trabajen de manera explícita. Este tipo de contenidos es también de naturaleza muy diversa y pueden referirse a estrategias metacognitivas (aquellas que se centran en la reflexión sobre el proceso), socioafectivas (relacionadas con la dimensión personal del aprendizaje y la manera en que cada individuo hace frente a los sentimientos que le genera, así como a la forma de relacionarse con el entorno educativo), estrategias para la mejora de la comunicación (suplir carencias, resultar más efectivos, compensar déficits...) o estrategias para el desarrollo de destrezas y para un mejor aprendizaje de los aspectos lingüísticos.

El trabajo de análisis y valoración concluye definitivamente con un apartado abierto de "Observaciones" en el que anotar impresiones generales, valoraciones críticas, aspectos de mejora, carencias, logros destacables, contextos y perfiles idóneos para su uso, correspondencia con los niveles de referencia europeo, grado aproximado de cumplimiento de los criterios desarrollados en los documentos educativos de mayor incidencia para la enseñanza del español - criterios que hemos venido revisando a lo largo de estas páginas -. En fin, se trata de espacio destinado tanto a las conclusiones del estudio como a suplir las propias carencias de la propuesta, por lo que animamos a que se complete y mejore añadiendo cuantos apartados y aspectos se consideren oportunos para cada caso.

A continuación, presentamos de modo esquemático la herramienta que acabamos de describir.

\section{Datos identificativos}

- Autor/es

- Título

- Datos bibliográficos

- Otras obras del autor/es 


\section{Aspectos formales}

\subsection{Material que lo compone}

\begin{tabular}{|l|l|l|l|l|l|}
\hline & Soporte & Calidad & Adecuación & Naturalidad & Funcionalidad \\
\hline Audio (sonoro) & & & & & \\
\hline Vídeo (visual) & & & & & \\
\hline Impreso - Portfolio & & & & & \\
\hline Impreso - Cuaderno & & & & & \\
\hline Impreso - Guía & & & & & \\
\hline CD ROM interactivo & & & & & \\
\hline Soporte web de apoyo & & & & & \\
\hline
\end{tabular}

\subsection{Edición (elementos de diseño)}

\begin{tabular}{|l|l|l|l|l|l|}
\hline & $\begin{array}{l}\text { De qué } \\
\text { tipo }\end{array}$ & Funcionalidad & Adecuación & $\begin{array}{l}\text { Imagen que se } \\
\text { transmite }\end{array}$ & $\begin{array}{l}\text { Diversidad } \\
\text { sociocultural }\end{array}$ \\
\hline Ilustraciones & & & & & \\
\hline Fotografías & & & & & \\
\hline Tipografía & & & & & \\
\hline Colores & & & & & \\
\hline Iconos & & & & & \\
\hline
\end{tabular}

\subsection{Estructura, elementos y organización}

\begin{tabular}{|c|c|c|c|c|c|c|c|c|}
\hline $\begin{array}{l}\text { Partes } \\
\text { que lo } \\
\text { componen }\end{array}$ & Presentación & $\begin{array}{l}\text { Cuadro de } \\
\text { programación }\end{array}$ & $\begin{array}{l}\mathrm{N}^{\circ} \text { de } \\
\text { unidades } \\
\text { didácticas }\end{array}$ & Transcripciones & Glosario & $\begin{array}{l}\text { Apéndice } \\
\text { gramatical }\end{array}$ & $\begin{array}{l}\text { Evaluación } \\
\text { o auto- } \\
\text {-evaluación }\end{array}$ & Otros \\
\hline & & & & & & & & \\
\hline
\end{tabular}




\begin{tabular}{|l|l|l|l|l|l|}
\hline $\begin{array}{l}\text { Estructura } \\
\text { de cada } \\
\text { unidad }\end{array}$ & $\begin{array}{l}\text { Sin apartados } \\
\text { (secuencia } \\
\text { de aprendizaje) }\end{array}$ & $\begin{array}{l}\text { Apartados } \\
\text { por } \\
\text { destrezas }\end{array}$ & $\begin{array}{l}\text { Apartados } \\
\text { por niveles } \\
\text { de lengua }\end{array}$ & $\begin{array}{l}\text { Apartados } \\
\text { por ámbitos } \\
\text { temáticos }\end{array}$ & $\begin{array}{l}\text { Lugar de la } \\
\text { información } \\
\text { gramatical, } \\
\text { funcional... }\end{array}$ \\
\cline { 2 - 6 } & & & & & \\
\hline
\end{tabular}

\begin{tabular}{|l|l|l|l|}
\hline $\begin{array}{l}\text { Secuenciación } \\
\text { de cada } \\
\text { apartado }\end{array}$ & $\begin{array}{l}\text { No existe una } \\
\text { secuenciación } \\
\text { determinada }\end{array}$ & $\begin{array}{l}\text { Existe el mismo tipo de } \\
\text { secuenciación en cada } \\
\text { apartado, que consiste en... }\end{array}$ & $\begin{array}{l}\text { Cada apartado } \\
\text { tiene un tipo de } \\
\text { secuenciación, } \\
\text { que consiste en... }\end{array}$ \\
\cline { 2 - 4 } & & & \\
\hline
\end{tabular}

\section{Situación educativa}

\subsection{Destinatarios}

\begin{tabular}{|c|c|c|c|c|c|}
\hline \multicolumn{3}{|l|}{ NIÑOS } & \multicolumn{3}{|l|}{ JÓVENES } \\
\hline 6 a 8 años & 8 a 10 años & 10 a 12 años & 12 a 14 años & 14 a 16 años & 16 a 18 años \\
\hline
\end{tabular}

\subsection{Nivel}

\begin{tabular}{|l|l|l|l|l|l|l|}
\hline Nivel 0 & Nivel A1 & Nivel A2 & Nivel B1 & Nivel B2 & Nivel C1 & Nivel C2 \\
\hline & & & & & & \\
& & & & & & \\
\hline
\end{tabular}

\subsection{Duración}

\begin{tabular}{|l|l|l|l|l|}
\hline Intensivo & Trimestral & Cuatrimestral & Semestral & Anual \\
\hline & & & & \\
& & & & \\
\hline
\end{tabular}




\subsection{Contexto educativo}

\begin{tabular}{|l|l|l|l|}
\hline \multicolumn{2}{|l|}{ Enseñanza reglada } & Enseñanza no reglada \\
\hline Primaria & Secundaria & Bachillerato & \\
\hline & & & \\
& & & \\
\hline
\end{tabular}

\subsection{Programa y situación educativa}

\begin{tabular}{|l|l|l|l|}
\hline $\begin{array}{l}\text { El español es } \\
\text { asignatura de } \\
\begin{array}{l}\text { lengua extranjera } \\
\text { (obligatoria u } \\
\text { opcional) }\end{array}\end{array}$ & $\begin{array}{l}\text { Programas bilingües } \\
\text { (algunas asignaturas } \\
\text { se imparten en } \\
\text { español) }\end{array}$ & $\begin{array}{l}\text { Español como } \\
\text { L2 en contextos } \\
\text { hispanohablantes }\end{array}$ & Otros \\
\hline & & & \\
\hline
\end{tabular}

\section{Objetivos generales}

\begin{tabular}{|c|c|c|c|c|c|c|c|}
\hline $\begin{array}{l}\text { El alumno } \\
\text { como agente }\end{array}$ & \multicolumn{3}{|c|}{ Transacciones } & \multicolumn{2}{|c|}{ Interacciones sociales } & \multicolumn{2}{|c|}{$\begin{array}{l}\text { Acciones con textos orales y } \\
\text { escritos }\end{array}$} \\
\hline $\begin{array}{l}\text { El alumno } \\
\text { como hablante } \\
\text { intercultural }\end{array}$ & $\begin{array}{l}\text { Diversidad } \\
\text { cultural }\end{array}$ & \multicolumn{2}{|c|}{$\begin{array}{l}\text { Actitudes } \\
\text { y factores } \\
\text { afectivos }\end{array}$} & $\begin{array}{l}\text { Referentes } \\
\text { culturales }\end{array}$ & $\begin{array}{l}\text { Normas y } \\
\text { convenciones } \\
\text { sociales }\end{array}$ & $\begin{array}{l}\text { Participación } \\
\text { en situaciones } \\
\text { interculturales }\end{array}$ & $\begin{array}{l}\text { Papel como } \\
\text { intermediario } \\
\text { cultural }\end{array}$ \\
\hline $\begin{array}{l}\text { El alumno } \\
\text { como } \\
\text { aprendiente }\end{array}$ & $\begin{array}{l}\text { Control del } \\
\text { proceso de } \\
\text { aprendizaje }\end{array}$ & $\begin{array}{l}\text { Plan } \\
\text { del }\end{array}$ & $\begin{array}{l}\text { ficación } \\
\text { prendizaje }\end{array}$ & $\begin{array}{l}\text { Gestión de } \\
\text { recursos }\end{array}$ & $\begin{array}{l}\text { Uso estratégico } \\
\text { procedimientos } \\
\text { de aprendizaje }\end{array}$ & $\begin{array}{l}\text { Control de } \\
\text { factores } \\
\text { socioafectivos }\end{array}$ & $\begin{array}{l}\text { Cooperación } \\
\text { con el grupo }\end{array}$ \\
\hline $\begin{array}{l}\text { El alumno } \\
\text { como } \\
\text { estudiante } \\
\text { de programa } \\
\text { reglado }\end{array}$ & \multicolumn{2}{|c|}{$\begin{array}{l}\text { Competencia social } \\
\text { y ciudadana }\end{array}$} & \multicolumn{2}{|c|}{$\begin{array}{l}\text { Competencia cultural y } \\
\text { artística }\end{array}$} & \multicolumn{2}{|l|}{$\begin{array}{l}\text { Competencia } \\
\text { medioambiental }\end{array}$} & $\begin{array}{l}\text { Competencia para } \\
\text { la salud }\end{array}$ \\
\hline
\end{tabular}




\section{Metodología}

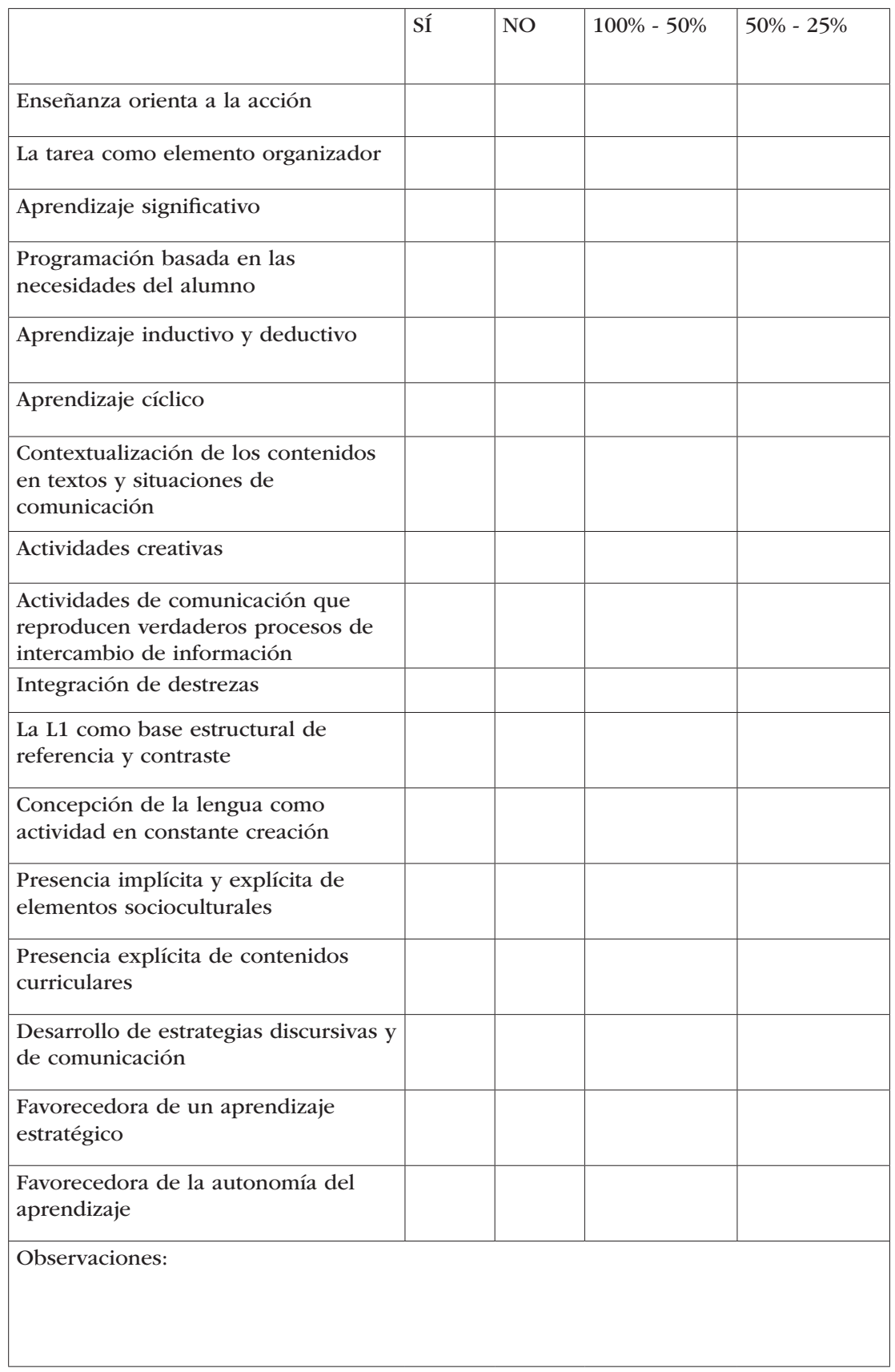




\section{Dinámicas y formas de trabajo}

\subsection{Tipología de actividades}

\begin{tabular}{|l|l|l|l|l|l|l|l|l|}
\hline $\begin{array}{l}\text { Presentación } \\
\text { de } \\
\text { contenidos }\end{array}$ & $\begin{array}{l}\text { Reflexión y } \\
\text { descubrimiento }\end{array}$ & Estructurales & $\begin{array}{l}\text { Comunicación } \\
\text { dirigida }\end{array}$ & $\begin{array}{l}\text { Comunicación } \\
\text { semidirigida }\end{array}$ & $\begin{array}{l}\text { Comunicación } \\
\text { libre }\end{array}$ & Juegos & Tareas & Otros \\
\hline & & & & & & & \\
\hline
\end{tabular}

\subsection{Dinámicas}

\begin{tabular}{|l|l|l|l|l|}
\hline & En algunas ocasiones & A menudo & Con mucha frecuencia & Constantemente \\
\hline $\begin{array}{l}\text { Presentaciones y } \\
\text { explicaciones }\end{array}$ & & & & \\
\hline Trabajo individual & & & & \\
\hline Trabajo en parejas & & & & \\
\hline Trabajo en grupos & & & & \\
\hline Trabajo conjunto & & & & \\
\hline Estudio & & & & \\
\hline Observaciones: & & & & \\
\end{tabular}

\section{Componentes}

\subsection{Enunciados}

\begin{tabular}{|l|l|}
\hline Tipos de enunciados & Características \\
\hline & \\
& \\
\hline
\end{tabular}


7.2. Textos escritos. Tipos

\begin{tabular}{|l|l|l|l|l|l|l|l|l|}
\hline Canciones & Poemas & Cuentos & $\begin{array}{l}\text { Adivinanzas, } \\
\text { refranes... }\end{array}$ & Diálogos & $\begin{array}{l}\text { Cartas, } \\
\text { postales, } \\
\text { notas }\end{array}$ & $\begin{array}{l}\text { Artículos } \\
\text { de prensa } \\
\text { o similar }\end{array}$ & $\begin{array}{l}\text { Textos de } \\
\text { divulgación }\end{array}$ & Otros \\
\hline & & & & & & & & \\
\hline
\end{tabular}

\subsection{Textos orales. Tipos}

\begin{tabular}{|l|l|l|l|l|l|l|}
\hline Canciones & Poemas & Cuentos & Diálogos & $\begin{array}{l}\text { Noticias, } \\
\text { anuncios }\end{array}$ & $\begin{array}{l}\text { Charlas, } \\
\text { conferencias, } \\
\text { explicaciones }\end{array}$ & Otros \\
\hline & & & & & & \\
\hline
\end{tabular}

\subsection{Textos escritos. Funcionalidad}

\begin{tabular}{|l|l|l|l|l|l|l|l|l|}
\hline $\begin{array}{l}\text { Introducción } \\
\text { de la unidad }\end{array}$ & $\begin{array}{l}\text { Presentación } \\
\text { de los } \\
\text { contenidos }\end{array}$ & $\begin{array}{l}\text { Contextualización } \\
\text { contenidos y } \\
\text { situaciones }\end{array}$ & $\begin{array}{l}\text { Trabajo de } \\
\text { contenidos } \\
\text { gramaticales } \\
\text { o léxicos }\end{array}$ & $\begin{array}{l}\text { Trabajo de } \\
\text { contenidos } \\
\text { funcionales }\end{array}$ & $\begin{array}{l}\text { Trabajo } \\
\text { de contenidos } \\
\text { socioculturales }\end{array}$ & $\begin{array}{l}\text { Desarrollo } \\
\text { de } \\
\text { destrezas } \\
\text { escritas }\end{array}$ & $\begin{array}{l}\text { Sin } \\
\text { funciones } \\
\text { específicas }\end{array}$ & Otros \\
\hline & & & & & & & \\
\hline
\end{tabular}

\subsection{Textos orales. Funcionalidad}

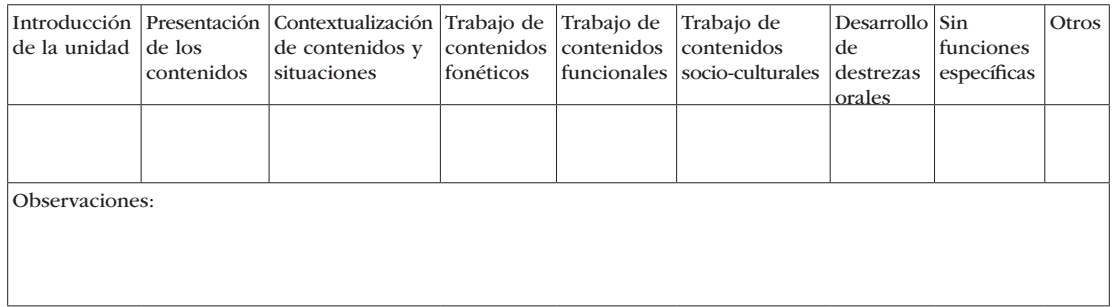




\subsection{Fichas}

\begin{tabular}{|l|l|l|l|}
\hline $\begin{array}{l}\text { Tipo de } \\
\text { información: } \\
\text { gramatical, léxica, } \\
\text { funcional, cultural... }\end{array}$ & $\begin{array}{l}\text { Frecuencia y } \\
\text { ubicación }\end{array}$ & Funcionalidades & Formato \\
\hline & & & \\
\hline
\end{tabular}

\section{Contenidos}

\subsection{Contenidos de comunicación}

\begin{tabular}{|l|l|l|l|}
\hline Tema & $\begin{array}{l}\text { Ámbitos nocionales } \\
\text { que se trabajan }\end{array}$ & $\begin{array}{l}\text { Situaciones de } \\
\text { comunicación e } \\
\text { interacción }\end{array}$ & $\begin{array}{l}\text { Funciones } \\
\text { comunicativas }\end{array}$ \\
\hline $1-$ & & & \\
\hline $2-$ & & & \\
\hline $3-$ & & & \\
\hline $4-$ & & & \\
\hline $5-$ & & & \\
\hline$\ldots$ & & & \\
\hline
\end{tabular}

\subsection{Contenidos lingüísticos}

\begin{tabular}{|l|l|l|}
\hline Fonéticos y ortológicos & Gramaticales & Léxicos (campos semánticos) \\
\hline & & \\
\hline
\end{tabular}

\subsection{Contenidos de otras áreas del currículo}

\begin{tabular}{|l|l|l|l|l|l|l|l|l|l|l|}
\hline Lengua & Matemáticas & $\begin{array}{l}\text { Conocimiento } \\
\text { del Medio }\end{array}$ & Literatura & Geografía & Historia & $\begin{array}{l}\text { Ciencias } \\
\text { naturales }\end{array}$ & Tecnología & $\begin{array}{l}\text { Física y } \\
\text { Química }\end{array}$ & $\begin{array}{l}\text { Educación } \\
\text { artística }\end{array}$ & $\begin{array}{l}\text { Educación } \\
\text { física }\end{array}$ \\
\hline & & & & & & & & & & \\
\hline
\end{tabular}




\subsection{Referentes culturales}

\begin{tabular}{|l|l|l|}
\hline $\begin{array}{l}\text { Conocimientos generales } \\
\text { de países hispanos }\end{array}$ & $\begin{array}{l}\text { Acontecimientos y } \\
\text { protagonistas del pasado y } \\
\text { del presente }\end{array}$ & $\begin{array}{l}\text { Productos y creaciones } \\
\text { culturales }\end{array}$ \\
\hline & & \\
\hline
\end{tabular}

\subsection{Saberes y comportamientos culturales}

\begin{tabular}{|l|l|l|}
\hline $\begin{array}{l}\text { Condiciones de vida y } \\
\text { organización social }\end{array}$ & Relaciones interpersonales & $\begin{array}{l}\text { Identidad colectiva y estilo } \\
\text { de vida }\end{array}$ \\
\hline & & \\
\hline
\end{tabular}

\subsection{Contenidos para un aprendizaje estratégico}

\begin{tabular}{|l|l|l|l|l|}
\hline $\begin{array}{l}\text { Estrategias } \\
\text { metacognitivas }\end{array}$ & $\begin{array}{l}\text { Estrategias } \\
\text { socioafectivas }\end{array}$ & $\begin{array}{l}\text { Estrategias } \\
\text { para la } \\
\text { mejora de la } \\
\text { comunicación }\end{array}$ & $\begin{array}{l}\text { Estrategias para } \\
\text { el desarrollo } \\
\text { destrezas } \\
\text { comunicativas }\end{array}$ & $\begin{array}{l}\text { Estrategias para } \\
\text { el aprendizaje } \\
\text { de los aspectos } \\
\text { lingüísticos }\end{array}$ \\
\hline & & & \\
\hline
\end{tabular}

\section{OBSERVACIONES:}

\section{CONCLUSIONES}

Ante las perspectivas de desarrollo del español como lengua extranjera en contextos escolares, creemos necesario diseñar instrumentos de análisis y de valoración del material didáctico existente que permita a los diferentes implicados en los procesos de enseñanza-aprendizaje detectar carencias, establecer criterios y guiar su elaboración y 
selección. Estos son los objetivos fundamentales de la propuesta que hemos descrito, propuesta que, a diferencia de otras formuladas con anterioridad - de las que, sin duda, es deudora -, se centra exclusivamente en enseñanza a niños y jóvenes, sector de la población en el que se prevé el aumento de demanda más significativo.

Los criterios expuestos a lo largo de estas páginas no responden a una perspectiva personal sobre la metodología de las lenguas extranjeras ni a preferencias didácticas o pedagógicas, sino que son el resultado de la revisión crítica de algunos documentos educativos de gran relevancia para la enseñanza del español, de los que hemos extraído aquellos aspectos de mayor interés o que están directamente relacionados con el caso que nos ocupa - los contextos escolares -. Se trata, por tanto, de una propuesta fundamentada y claramente orientada a satisfacer unas necesidades - las de los centros escolares - y a promover un aprendizaje acorde con las directrices institucionales y oficiales actuales.

Dado el escaso material didáctico existente para este tipo de alumnado y lo alejado que dicho material está de los criterios aquí manejados, nuestra herramienta de análisis no ha podido ser experimentada y validada con la suficiente amplitud ${ }^{5}$, de ahí que no podamos valorar sus limitaciones y las necesidades de reajuste que presenta. Sin duda, para comprobar el cumplimiento de nuestros objetivos y expectativas - y hacer de ella una herramienta de trabajo útil y productiva - sería conveniente que fuera sometida a prueba por autores, editores, profesores, gestores educativos y expertos en la materia de diferentes países, con realidades educativas también distintas.

En el momento actual de innovación educativa y de cambios transcendentales en las bases psicopedagógicas de la enseñanza

${ }^{5}$ La propuesta ha sido probada con la mayor parte de los materiales destinados a niños y jóvenes publicados en España y con algunos de otros países (Brasil, Reino Unido, Alemania). Debo agradecer este trabajo a muchos de mis alumnos del máster de enseñanza de español de los dos últimos años, que, voluntariamente, han querido colaborar conmigo. 
(aprendizaje experiencial y por competencias, aprendizaje constructivista e integrador, enseñanza centrada en el alumno, autonomía y aprendizaje estratégico, diversidad y estilos de aprendizaje...) la elaboración de material didáctico no puede continuar siendo una labor más o menos individual, en la que el autor/profesor interpreta libremente las nuevas orientaciones y, libremente también las incorpora o no, siempre desde su perspectiva. Debe contar con herramientas que orienten de forma clara y precisa esa labor de creación, que puedan aplicarse inequívocamente. Para que ello sea posible, se requiere de trabajos que investiguen el impacto de todos estos nuevos planteamientos en los aprendizajes, así como la manera de incorporarlos al material didáctico (editado o no), y que propongan líneas de actuación y orientaciones concretas, no de carácter teórico, sino a través de instrumentos de análisis y de desarrollo verdaderamente útiles. En este sentido, queda todavía mucho por hacer, y nuestra propuesta es solo un pequeño paso.

\section{B I B LI O G RA FíA}

Agercel. (2000). Quality guide for the evaluation and design of language learning and teaching programmes and materials [CD-ROM]. Bruselas: EUROCENTRES. [Documento de Internet disponible en www.agercel.com].

Areizaga Orube, E. (1997). Dos décadas de enseñanza del español como lengua extranjera a adultos en sus materiales. Bilbao: Servicio Editorial de la Universidad del País Vasco.

Azevedo, M. M. (1978). Trends in Elementary Spanish Texts. The Modern Language Journal, 62, 399-407.

Consejo de Europa. (2005). Reference Level Descriptions for National and Regional Languages (RLD). Draft Guide for the production of RLD (Version 2). Estrasburgo: Language Policy Division, DG IV.

Consejo Social Europeo. (2002). Marco común europeo de referencia para las lenguas: aprendizaje, enseñanza, evaluación. Madrid: MECD e Instituto Cervantes.

Ezeiza Ramos, J. (2008). Analizar y comprender la topografía configuracional de los materiales de enseñanza de lenguas en una perspectiva de síntesis. Aplicación a los manuales para la enseñanza general de E/LE a adultos. Madrid: Servicio de Publicaciones de la Universidad Antonio de Nebrija. 
Ezquerra, R. (1974). Análisis de métodos para la enseñanza del español. Boletín de la Asociación Europea de Profesores de Español (AEPE), 11, 31-46.

Fenner, A. \& Newby, D. (2000). Approaches to materials design in european textbooks: implementing principles of authenticity, learner autonomy and cultural awareness. Austria: Consejo de Europa.

Fenner, A. \& Newby, D. (2007). Coherence of principles, cohesion of competences Exploring theories and designing materials for teacher education. Austria: Consejo de Europa.

Fernández López, C. (2004). Principios y criterios para el análisis de materiales didácticos. In J. Sánchez Lobato \& I. Santos Gargallo (Dirs.), Vademécum para la formación de profesores (715-734). Madrid: SGEL.

Hopkins, A. (2000). Users' guide for textbook and materials writers. In S. Baillo, S. Devitt, M ${ }^{a}$ J. Gremio, F. Heyworth, A. Hopkins, B. Jones, M. Makosh, P. Riley, G. Stoks \& J. Trim (Eds.), Common European Framework of Reference for languages: learning, teaching, assessment. A guide for users (199-232). Estrasburgo: Language Policy Division.

Instituto Cervantes. (2006a.) Enciclopedia del español en el mundo. Madrid: Círculo de Lectores, IT \& Plaza y Janés.

Instituto Cervantes. (2006b.) Plan Curricular del Instituto Cervantes. Niveles de referencia para el español. Madrid: Instituto Cervantes - Biblioteca Nueva.

National Standards in Foreign Language Education Project. (1996). Standards for foreign language learning: Preparing for the 21 st century. Yonkers, NY: ACTFL.

Orientaçiões curriculares para o ensino médio. Conhecimientos de lengua estrangeira (espanhol). (2006). Brasilia: Secretaria de Educação Básica, Departamento de Politicas de Ensino Médio.

Ramírez, A. G. \& Hall, J. K. (1990). Language and Culture in Secondary Level Spanish Textbooks. The Modern Language Journal, 74 (I), 48-65.

Salaberri Ramiro, S. (1990). El libro de texto: selección y explotación. In P. Bello (Ed.), Didáctica de segundas lenguas. Estrategias y discursos básicos (109-123). Madrid: Santillana.

Spanos, G. (1989). On the integration of Language and Content Instruction. Annual Review of Applied Linguistics, 10, 227-240.

Trujillo, F. (2005). La integración de lenguaje y contenidos en la enseñanza del español como segunda lengua en el contexto escolar: implementación y evaluación. RedELE, 4. [Documento de Internet disponible en http://www.educacion.es/ redele/revista4/trujillo.shtml]. 